\title{
Review: cognitive behaviour therapy and behaviour therapy may be effective for back pain and chronic fatigue syndrome, and antidepressants may be effective for irritable bowel syndrome
}

\author{
Raine R, Haines A, Sensky T, et al. Systematic review of mental health interventions for patients with common somatic \\ symptoms: can research evidence from secondary care be extrapolated to primary care? BMJ 2002;325:1082-5. \\ QUESTION: In patients with 3 common somatic conditions (chronic fatigue syndrome, \\ irritable bowel syndrome, and chronic back pain), what is the effectiveness of mental \\ health interventions?
}

\section{Data sources}

English language studies published between 1966 and September 2001 were identified by searching PubMed, the Cochrane Library, PsycLIT, and EMBASE/Excerpta Medica. Bibliographies of relevant studies and reviews were scanned, and experts were contacted.

\section{Study selection}

Studies were selected if they were randomised controlled trials (RCTs) of patients with chronic fatigue syndrome, irritable bowel syndrome, or chronic back pain for which no physical cause could be found.

\section{Data extraction}

Data were extracted on study quality, patient characteristics, interventions, outcomes, dropouts, and reasons for withdrawal.

\section{Main results}

61 RCTs were identified; 20 were classified as primary care and 41 as secondary care. For patients with chronic back pain, cognitive behaviour therapy (7 studies in primary care [891 patients] and 9 in secondary care [625 patients]) showed improvements in pain, disability, and depression. Behaviour therapy (8 of 9 studies [659 patients] in primary care and 5 of 6 studies [398 patients] in secondary care) reported improvements in symptoms, and some evidence showed that improvements were sustained at 1 year follow up. No studies were identified on brief psychodynamic interpersonal therapy, and 2 studies of antidepressants gave insufficient data for drawing conclusions.

For patients with chronic fatigue syndrome, 4 studies on antidepressants showed no evidence for sustained symptomatic improvement. No studies were identified on brief dynamic psychotherapy. The effectiveness of cognitive behaviour therapy ( 5 studies) and behaviour therapy (3 studies) is uncertain in primary care, but evidence from secondary care seems to suggest positive effects.

For patients with irritable bowel syndrome, 12 studies suggest that antidepressants may be effective in relieving pain. 2 studies of brief dynamic psychotherapy showed some evidence of effectiveness in refractory patients. Cognitive behaviour therapy (3 studies [169 patients] in primary care and 5 studies [171 patients] in secondary care) showed mixed results for symptomatic improvement. The effectiveness of behaviour therapy (4 studies) is uncertain because of limited evidence.

\section{Conclusions}

For patients with chronic back pain or chronic fatigue syndrome, cognitive behaviour therapy and behaviour therapy may be effective. Antidepressants seem effective for patients with irritable bowel syndrome, but ineffective for patients with chronic fatigue syndrome.

\section{COMIMENTARY}

The study by Raine $e t$ al raises some important issues about clinical pathways for these common somatic problems. For example, if effective interventions are available, how do patients (and clinicians) find the way to them? Is primary care the most appropriate setting in which to deliver these interventions?

The authors propose several reasons for the differences in outcome between primary and secondary care settings. One difference relates specifically to back pain where a greater severity of disorder was found in secondary care. This may reflect the process whereby patients are referred to psychological care only when all other options are exhausted. Thus, the process may select out those whose pain fails to respond to medical treatments. But it may also mean that because every other option has been attempted, the patient is more receptive to a psychological intervention.

The beliefs of the patient about the causes of what is experienced as a physical problem will affect the uptake of psychological treatment. This is likely to be mediated by messages and attitudes transmitted through primary care and specialist care. For example, in a large multisite intervention trial for chronic fatigue syndrome, ${ }^{\text {t }}$ the authors suggest that relatively high refusal and withdrawal rates (almost 50\%) reflect the preconceptions of patients, who expected a somatically oriented treatment. Furthermore, therapists' attitudes about patients who have somatic presentations may also affect outcome. In the same trial, $82 \%$ of therapists believed that patients with chronic fatigue syndrome were more difficult to treat than those with psychiatric disorders. Raine $e t$ al also point to differences in the quality of care in primary and secondary care. For example, primary care based interventions have fewer contact hours. Good reasons may exist for the provision of briefer interventions in primary care, but complex problems require complex solutions. Complex solutions take time and require skill and resources that may not be available in primary care. It is unclear whether primary care personnel are willing or able to provide the level of care needed in the psychological treatment of somatic problems. Justin Kenardy, PhD University of Queensland Brisbane, Queensland, Australia

1 Prins JB, Bleijenberg G, Bazelmans E, et al. Cognitive behavine syndrome: a multi-centre randomised controlled trial. Lancet 2001;357:841-7. 